\title{
CrimRxiv
}

\section{Performance-Enhancing Substance Use and Intimate Partner Violence: A Prospective Cohort Study}

Kyle T. Ganson, Dylan B. Jackson, Alexander Testa, Jason M. Nagata

Published on: Feb 05, 2022

DOI: $10.21428 / \mathrm{cb} 6 \mathrm{ab} 371.883525 \mathrm{~d} 1$

License: Creative Commons Attribution 4.0 International License (CC-BY 4.0). 
